\title{
OSCILLATION THEOREMS FOR SYSTEMS OF LINEAR DIFFERENTIAL EQUATIONS
}

\author{
BY \\ ZEEV NEHARI $\left({ }^{1}\right)$
}

1. The systems to be considered in this paper are of the form

$$
y^{\prime}=A y,
$$

where $A=A(x)$ is a continuous $n \times n$ matrix on an $x$-interval $R$, and $y$ is an $n$ dimensional column vector. We shall assume that the elements of $A$ are real, and we shall consider only real solution vectors of (1.1). This is not an essential restriction since, in the complex case, (1.1) can be replaced by an equivalent real system with a $2 n \times 2 n$ coefficient matrix.

We shall say that a nontrivial solution vector $y=\left(y_{1}, \ldots, y_{n}\right)$ of $(1.1)$ is oscillatory on $R$ if each of its components takes the value zero at some point of $R$, i.e., $y_{k}\left(x_{k}\right)=0, x_{k} \in R, k=1, \ldots, n$. The system (1.1) itself will be said to be oscillatory if it possesses at least one oscillatory solution vector. If there is no such solution vector, i.e., if every nontrivial solution vector has a component which does not vanish on $R$, the system will be said to be nonoscillatory on $R$.

In a recent paper by B. Schwarz [16], systems with the latter property are called "disconjugate", rather than "nonoscillatory", and a word of justification for this change of terminology is in order. The term "disconjugate", as introduced by Wintner [21], refers to the absence of a conjugate point in the sense of Jacobi, and thus originally applied only to selfadjoint equations and systems [3], [4], [14], [15], [19], [20]. However, this concept generalizes in a natural way to general $n$th order differential equations [1], [8], [9], [10], [13], [17], [18] and thus also to systems which are equivalent to such equations. In all these cases, the right conjugate point $\eta\left(x_{0}\right)$ of $x_{0}\left(\eta\left(x_{0}\right)>x_{0}\right)$ is a continuous function of $x_{0}$, and the left conjugate point of $\eta\left(x_{0}\right)$ coincides with $x_{0}$ [17], [18]. In the case of a system which can be reduced to an $n$th order equation, $\eta\left(x_{0}\right)$ can be defined in the following way: there exists a solution vector of (1.1) such that every component of $y$ vanishes either at $x_{0}$ or at $\eta\left(x_{0}\right)$, and $\eta\left(x_{0}\right)$ is the smallest number with this property. It can then be shown that $\eta\left(x_{0}\right)=\inf b$, where $b$ is such that the system is oscillatory in $\left[x_{0}, b\right)$ [9], [13], [17].

In the case of a general system (1.1), the conjugate point may be defined in the same way, but it is in general not true that $\eta\left(x_{0}\right)=\xi\left(x_{0}\right)$, where $\xi\left(x_{0}\right)=\inf b$, and

Received by the editors February 6, 1968 and, in revised form, August 21, 1968.

( $\left.{ }^{1}\right)$ Research sponsored by the Air Force Office of Scientific Research, Office of Aerospace Research, United States Air Force, under AFOSR Grant No. 62-414. 
$\left[x_{0}, b\right)$ is an interval of oscillation of the system. That this can happen even in the case of a $2 \times 2$ matrix $A$, is shown by the following simple example. If

$$
A=\left(\begin{array}{ll}
\sigma & \sigma \\
\tau & \tau
\end{array}\right), \quad \sigma=\frac{2 x}{x^{2}+(x-1)^{2}}, \quad \tau=\frac{2(x-1)}{x^{2}+(x-1)^{2}},
$$

equation (1.1) has the two independent solution vectors $\left(x^{2},(x-1)^{2}\right)$ and $(1,-1)$. The general solution is thus $\left(\alpha x^{2}+\beta, \alpha(x-1)^{2}-\beta\right)$, where $\alpha, \beta$ are constants, and it is easily seen that $\left(x^{2},(x-1)^{2}\right)$ is the only oscillatory solution of the system. Accordingly, the point $x=1$ is the only point which possesses a conjugate point. On the other hand, $\xi\left(x_{0}\right)=1$ for all nonpositive $x_{0}$. Moreover, if we set $\xi\left(x_{0}\right)=\sup a$, where the system is oscillatory in $\left(a, x_{0}\right]$, we have $\xi\left(x_{0}\right)=0$ for all $x \geqq 1$, and this shows that $\xi\left[\xi\left(x_{0}\right)\right]=1 \neq x_{0}$ if $x_{0}>1$. It would therefore hardly be appropriate to call $\xi\left(x_{0}\right)$ the conjugate point of $x_{0}$. Accordingly, it seems preferable to say that the system is nonoscillatory in $\left(x_{0}, \eta\left(x_{0}\right)\right)$, rather than disconjugate.

We introduce here yet another concept which is closely related to nonoscillation, and which has the merit that it can be defined without reference to the components of the solution vectors. We shall say that the system (1.1) is suborthogonal on $R$ if, for any nontrivial solution vector $y$, and for any $s \in R, t \in R$,

$$
y(s) y(t)>0 .
$$

In the case of a $2 \times 2$ matrix, suborthogonality implies nonoscillation; indeed, if the two components of $y$ vanish at $s$ and $t$, respectively, we evidently have $y(s) y(t)=0$.

It may be noted that, if $C$ is a constant orthogonal matrix the system

$$
w^{\prime}=C A C^{-1} w
$$

is suborthogonal if the same is true of the system (1.1). Indeed, the general solution of (1.3) is of the form $C y$, where $y$ is the general solution of (1.1), and the assertion follows from the fact that $[C y(s)][C y(t)]=y(s) y(t)$. Nonoscillation is in general not preserved if the coefficient matrix $A$ is replaced by $C A C^{-1}$. However-and this points up the close relation between the concepts of nonoscillation and suborthogonality-if the system (1.3) is nonoscillatory on $R$ for all constant orthogonal matrices $C$, then it is also suborthogonal on this interval. To establish this assertion, suppose (1.1) has a nontrivial solution vector $y$ for which $y(s) y(t)=0, s \in R, t \in R$. If we determine the constant orthogonal matrix $C$ so that the vector $C y(s)$ has the components $(\|y(s)\| 0, \ldots, 0)$, it follows from $0=y(s) y(t)=[C y(s)][C y(t)]$ that the first component of $C y(t)$ is zero. Hence, (1.3) has a solution vector $w=C y$ all of whose components vanish at either $s$ or $t$ and it follows that (1.3) is oscillatory.

The suborthogonality of the system (1.1) can also be expressed in terms of a fundamental (i.e., nonsingular) solution matrix $Y$ of the matrix-matrix equation

$$
Y^{\prime}=A Y
$$

corresponding to (1.1). Since the general solution of (1.1) is of the form $y=Y \alpha$, where $\alpha$ is an arbitrary constant vector, condition (1.2) is equivalent to $\alpha Y^{*}(s) Y(t) \alpha$ 
$>0$, i.e., to the condition that the symmetric part of the matrix $Y^{*}(s) Y(t)$ be positive-definite for all $s \in R, t \in R$. In particular, if we choose a fundamental solution $Y_{s}$ of (1.3) which reduces to the unit matrix $I$ for $x=s$, the condition becomes $\alpha Y_{s}(t) \alpha>0$. With the help of this version of condition (1.2) it is easy to establish the following property of suborthogonal systems.

If the system (1.1) is suborthogonal, so is the adjoint system

$$
w^{\prime}=-A^{*} w
$$

Indeed, if the matrix $W_{s}(x)$ is the fundamental solution (with $W_{s}(s)=I$ ) of the matrix-matrix equation corresponding to (1.5), we have

$$
\left(W_{s}^{*} Y_{s}\right)^{\prime}=-W^{*} A Y+W^{*} A Y=0
$$

and therefore $W_{s}^{*} Y_{s}=I$. Hence, if $\beta$ is an arbitrary constant vector, and we set $\alpha=Y_{s}^{-1}(t) \beta=W_{s}^{*}(t) \beta$, we have $\beta W_{s}(t) \beta=\beta W_{s}^{*}(t) \beta=\alpha Y_{s}(t) \alpha>0$. Since $s, t$ and the constant vector $\beta$ were arbitrary, the assertion follows.

2. The principal aim of this paper is to obtain conditions-expressed in terms of the coefficient matrix-which guarantee the nonoscillation of the system (1.1) on a given interval. All these conditions will follow from two basic inequalities, which we state here in the form of a theorem.

THEOREM 2.1. Let $y$ and $w$ be nontrivial solution vectors of the systems:

$$
\begin{aligned}
& y^{\prime}=A y, \\
& w^{\prime}=B w,
\end{aligned}
$$

respectively, where the $n \times n$ matrices $A, B$ are continuous on the interval $[a, b]$. If $u, v$ are the unit vectors

$$
u=y /\|y\|, \quad v=w /\|w\|
$$

and $C$ is an arbitrary constant orthogonal matrix, then

$$
|\arcsin [u(b) C v(b)]-\arcsin [u(a) C v(a)]| \leqq \int_{a}^{b}(\|A\|+\|B\|) d x,
$$

where $\|A\|$ denotes the norm $\sup _{\|\alpha\|=1}\|A \alpha\|$.

If one of the systems (2.1), (2.2) is oscillatory on $[a, b],(2.4)$ can be replaced by the stronger inequality

$$
|\arcsin [u(b) C v(b)]|+|\arcsin [u(a) C v(a)]| \leqq \int_{a}^{b}(\|A\|+\|B\|) d x .
$$

Proof. Differentiating (2.3), we obtain

$$
u^{\prime}=y^{\prime} /\|y\|-y\left(y y^{\prime}\right) /\|y\|^{3},
$$

and a similar expression for $v^{\prime}$. In view of (2.1) and (2.2), this leads to

$$
u^{\prime}=A u-u(u A u)
$$

$$
v^{\prime}=B v-v(v B v)
$$


If $C$ is a constant orthogonal matrix, we have $(u C v)^{\prime}=u^{\prime} C v+v^{\prime} C^{*} u$ and thus, by (2.6) and (2.7),

$$
(u C v)^{\prime}=[C v-(u C v) u] A u+\left[C^{*} u-(u C v) v\right] B v .
$$

Hence, since $u$ and $v$ are unit vectors,

$$
\left|(u C v)^{\prime}\right| \leqq\|C v-(u C v) u\| \cdot\|A\|+\left\|C^{*} u-(u C v) v\right\| \cdot\|B\| .
$$

Because of

$$
\|C v-(u C v) u\|^{2}=\|C v\|^{2}-(u C v)^{2}=1-(u C v)^{2}
$$

and

$$
\left\|C^{*} u-(u C v) v\right\|^{2}=\left\|C^{*} u\right\|^{2}-(u C v)^{2}=1-(u C v)^{2}
$$

this implies

$$
\frac{(u C v)^{\prime}}{\left(1-(u C v)^{2}\right)^{1 / 2}} \leqq\|A\|+\|B\|
$$

and an integration establishes (2.4).

We now turn to the proof of inequality (2.5). Since $u C v=v C^{*} u$, we may assume without loss of generality that $w$ (and thus also $v$ ) is oscillatory on $[a, b]$. If $v_{1}, \ldots, v_{n}$ are the components of $v$, there will thus exist a set of points $x_{1}, \ldots, x_{n}$ in $[a, b]$, containing at least two different points (since otherwise $w$ would reduce to the trivial solution), such that $v_{k}\left(x_{k}\right)=0, k=1, \ldots, n$. Evidently, the vector $C v\left(x_{k}\right)$ is not changed if the elements $c_{i k}, i=1, \ldots, n$ in the $k$ th column of the matrix $C$ are replaced by different numbers. We shall take advantage of this fact by substituting $-c_{i k}$ for $c_{i k}(i=1, \ldots, n)$, and we note that this change does not affect the orthogonal character of the matrix. Proceeding from $a$ to $b$, and making this change whenever a point $x_{k}$ is crossed, we obtain a matrix function $C(x)$ which is constant and orthogonal in the intervals between adjacent points $x_{k}$. By the construction of $C(x)$, the vector function $C(x) v(x)$ is continuous on $[a, b]$, and we evidently have $C(b)=-C(a)=-C$.

In any interval between adjacent points $x_{k}$ we may use (2.9) with $C(x)$ substituted for $C$. We integrate, and add up the contributions from all the intervals making up $[a, b]$. Since $C(x) v(x)$ is continuous, and since $C(b)=-C(a)=-C$, we obtain

$$
|\arcsin [u(b) C v(b)]+\arcsin [u(a) C v(a)]| \leqq \int_{a}^{b}(\|A\|+\|B\|) d x .
$$

Combining this with (2.4), we obtain (2.5). It is easy to see that this argument remains valid if some (or even all) of the points $x_{k}$ coincide with either $a$ or $b$.

In the special case in which $C$ is a diagonal matrix whose elements $c_{k k}$ are either 1 or -1 , the continuity of $C(x)$ is not affected by changing $c_{k k}$ into $-c_{k k}$ at a point at which the $k$ th component of either $u$ or $v$ is zero. In order to obtain inequality (2.5) it is therefore sufficient to assume that, for each $k(k=1, \ldots, n)$, 
the $k$ th component of at least one of the vectors $y, w$ vanishes on $[a, b]$. If, for $x=a$, we take $C$ to be the unit matrix, this leads to the following result.

THEOREM 2.2. Let $y, w, A, B, u, v$ have the same meaning as in Theorem (2.1). If, for each $k(k=1, \ldots, n)$, the kth component of at least one of the vectors $y, w$ vanishes at a point of $[a, b]$, then

$$
|\arcsin [u(b) v(b)]|+|\arcsin [u(a) v(a)]| \leqq \int_{a}^{b}(\|A\|+\|B\|) d x .
$$

3. As a first application of Theorem 2.1 we derive the following sufficient condition for suborthogonality.

THEOREM 3.1. If, for some continuous real function $\mu=\mu(x)$ on $[a, b]$, we have

$$
\int_{a}^{b}\|A+\mu I\| d x<\frac{\pi}{2}
$$

then the system (2.1) is suborthogonal on $[a, b]$. The constant $\pi / 2$ in (3.1) is the best possible; in fact, the conclusion does not necessarily hold if the sign of equality is permitted in (3.1).

Proof. We use (2.4), with $B=0$ and $C=I$. Since (2.2) is solved by an arbitrary constant vector, $v$ may be taken to be an arbitrary constant unit vector. If $a \leqq s$ $<t \leqq b$, and we set $v=u(t),(2.4)$ becomes

$$
\left|\frac{\pi}{2}-\arcsin [u(s) u(t)]\right| \leqq \int_{s}^{t}\|A\| d x \leqq \int_{a}^{b}\|A\| d x .
$$

If (2.1) is not suborthogonal on $[a, b]$, there exist $s, t \in[a, b]$ such that $u(s) u(t)=0$. In this case we thus must have $\pi / 2 \leqq \int_{a}^{b}\|A\| d x$. If $\mu \equiv 0$, this conflicts with (3.1) and thus proves Theorem (3.1) in this particular case. The case of a general continuous function $\mu$ is easily reduced to the case $\mu=0$, since the general solution of the system $\sigma^{\prime}=\left(A+I_{\mu}\right) \sigma$ is of the form $\sigma=g y$, where $g(x)$ is the scalar function $\exp \left\{\int^{x} \mu d x\right\}$ and $y$ is the general solution of (2.1). We have $\sigma(s) \sigma(t)=g(s) g(t)$ $\times[y(s) y(t)]$ and, since $g \neq 0$, the system $\sigma^{\prime}=(A+\mu I) \sigma$ is suborthogonal if, and only if, the same is true of the system (2.1).

To show that the constant in Theorem 3.1 is the best possible, we set $n=2 m$, where $m$ is a positive integer, and we consider the coefficient matrix $A$ whose elements $a_{i k}$ are defined as follows: $a_{k, k+1}=1, k=1, \ldots, n-1, a_{n, 1}=(-1)^{m}$; all other elements of $A$ are zero. It is easily confirmed that the system (2.1) associated with this matrix has a solution vector $y=\left(y_{1}, \ldots, y_{2 m}\right)$ with $y_{2 k+1}=(-1)^{k} \sin x$, $k=0, \ldots, m-1$, and $y_{2 k}=(-1)^{k+1} \cos x, k=1, \ldots, m$. Accordingly, we have $y(0) y(\pi / 2)=0$, i.e., the system is not suborthogonal on $[0, \pi / 2]$. On the other hand, it is easily confirmed that $\|A\|=1$, and thus

$$
\int_{0}^{\pi / 2}\|A\| d x=\frac{\pi}{2}
$$


This shows that (3.1) (with the particular choice $\mu=0$ ) is the best possible condition of its kind and that suborthogonality does not necessarily obtain if the sign of equality holds in (3.1). We also note for further reference that the exhibited solution vector is oscillatory on $[0, \pi / 2]$.

Turning now to criteria for nonoscillation, we set $B=0, C=I$ in (2.5) and, as before, we identify the arbitrary constant unit vector $v$ with $u(a)$. An application of Theorem 2.1 then leads to the following result.

THEOREM 3.2. If the solution vector $y$ of $(2.1)$ is oscillatory on $[a, b]$, then

$$
\frac{\pi}{2}+\arcsin \frac{|y(a) y(b)|}{\|y(a)\|\|y(b)\|} \leqq \int_{a}^{b}\|A\| d x .
$$

As an immediate corollary of this result we find that the condition $\int_{a}^{b}\|A\| d x$ $<\pi / 2$ is sufficient to guarantee the nonoscillation of the system $(2.1)$ on $[a, b]$. However, this criterion can be given a more general form with the help of an arbitrary diagonal $n \times n$ matrix $P$, whose diagonal elements $p_{k k}$ are continuously differentiable and do not vanish on $[a, b]$. If $w=P y$ and $y$ is a solution of (2.1), the vector $w$ is a solution of

$$
w^{\prime}=\left(P A P^{-1}+P^{\prime} P^{-1}\right) w
$$

and, as remarked by B. Schwarz [16], the system (3.3) is nonoscillatory on an interval if and only if the same is true of the system 2.1 ; indeed, if $y_{k}$ and $w_{k}$ are the components of $y$ and $w$, respectively, then $w_{k}=p_{k k} y_{k}$, and $p_{k k} \neq 0$. We thus have the following result.

THEOREM 3.3. Let $P$ be a diagonal $n \times n$ matrix whose diagonal elements are continuously differentiable and do not vanish on $[a, b]$, and let $A$ be a continuous $n \times n$ matrix on this interval. If

$$
\int_{a}^{b}\left\|P A P^{-1}+P^{\prime} P^{-1}\right\| d x<\frac{\pi}{2}
$$

then the system (2.1) is nonoscillatory on $[a, b]$. The constant $\pi / 2$ in (3.4) is the best possible, and the conclusion does not necessarily hold if the sign of equality is permitted in (3.4).

A weaker form of condition (3.4) (with the constant 1 instead of $\pi / 2$ ) was recently obtained by W. J. Kim [7]. (For nonoscillation criteria of a different type see [11], [12], [16].) The sharpness of (3.4) can be verified (for $P=I$ ), with the help of the same example which was used to show that Theorem 3.1 is the best possible of its kind.

4. The presence of the $n$ arbitrary functions in the main diagonal of $P$ lends a great deal of flexibility to condition (3.4). For a given $A$, the best choice of $P$ would be that which minimizes the integral on the left-hand side (and thus increases the interval to which the condition may be applied). Since the resulting variational problem will in general present great technical difficulties, it will often be more 
rewarding to choose a matrix $P$ of simple type which depends on some arbitrary parameters, and then to find the best criterion obtainable in this way.

We shall illustrate this method in the case of a system (2.1) which is equivalent to the $n$th order scalar differential equation

$$
\sigma^{(n)}+r(x) \sigma=0,
$$

where $v(x)$ is continuous on the interval $[a, b]$. If $A$ is the $n \times n$ matrix whose only nonzero elements $a_{i k}$ are $a_{k, k+1}=1, k=1, \ldots, n-1$ and $a_{n 1}=-r$, the solution vector $y$ of $(2.1)$ has the components $\sigma, \sigma^{\prime}, \ldots, \sigma^{(n-1)}$, where $\sigma$ is the solution of (4.1). The nonoscillation of the system is thus equivalent to the condition that, for any solution $\sigma$ of (4.1), at least one of the functions $\sigma, \sigma^{\prime}, \ldots, \sigma^{(n-1)}$ does not vanish in the interval in question. An equation with this property is said to be disfocal on the interval [13]. It may be noted that, by Rolle's theorem, a real disfocal equation is a fortiori disconjugate, i.e., none of its solutions can have more than $n-1$ zeros on the interval.

THEOREM 4.1. Let $R$ be a closed $x$-interval and let $S$ be a measurable subset of $R$ of Lebesgue measure $\mu(S)$. If $r(x)$ is continuous on $R$, if $r(x) \neq 0$ except on a null set, and if

$$
\sup _{S}[\mu(S)]^{n-1} \int_{R-S}|r| d x<(n-1)^{n-1}\left(\frac{\pi}{2 n}\right)^{n},
$$

then equation (4.1) is disfocal on $R$. The constant in (4.2) is the best possible.

If $r$ is of constant sign and $|r|$ is monotonic on $R$, the expression (4.2) can be simplified. For instance, if $|r|$ is nondecreasing and $R$ is the interval $[a, b],(4.2)$ may evidently be replaced by the condition

$$
\sup _{c}(c-a)^{n-1} \int_{c}^{b}|r| d x<(n-1)^{n-1}\left(\frac{\pi}{2 n}\right)^{n}, \quad c \in[a, b] .
$$

To prove Theorem 4.1, we use a constant diagonal matrix $P$. If we set $p_{k k}=\beta^{n-k}$, $k=1, \ldots, n$, where $\beta$ is a positive constant, the matrix $P A P^{-1}=\left(b_{i k}\right)$ has the elements $b_{k, k+1}=\beta, k=1, \ldots, n-1, b_{n, 1}=-r \beta^{-n+1}$, and all the other elements $b_{i k}$ are zero. It is easy to see that

$$
\left\|P A P^{-1}+P^{\prime} P^{-1}\right\|=\left\|P A P^{-1}\right\|=\max \left[\beta,|r| \beta^{-n+1}\right],
$$

and we may therefore conclude from Theorem 3.3 that the system associated with the coefficient matrix $A$ is nonoscillatory on $R$ (and, therefore, the equation (4.1) is disfocal on $R$ ) if

$$
\int_{R}\left\{\max \left[\beta,|r| \beta^{-n+1}\right]\right\} d x<\frac{\pi}{2} .
$$

If $S$ denotes the subset of $R$ on which $|r| \leqq \beta^{n}$, this may be written in the form

$$
\beta \mu(S)+\beta^{-n+1} \int_{R-S}|r| d x<\frac{\pi}{2}
$$


A simple approximation argument shows that it is sufficient to treat the case in which $r$ is not constant on any subinterval of $R$. In this case it is possible to choose $\beta$ in such a way that

$$
\frac{\beta^{n}}{n-1}=\frac{\int_{R-S}|r| d x}{\mu(S)}
$$

Indeed, the set $S$ depends on $\beta$, and it is easy to see that the right-hand side of (4.5) varies continuously from 0 to $\infty$ if $\beta^{n}$ decreases from $\max |r|$ to 0 . Hence, there must exist a positive $\beta$ for which (4.5) holds. For this particular value of $\beta$, the left-hand side of (4.4) takes the form

$$
\frac{n}{n-1}\left\{(n-1)[\mu(S)]^{n-1} \int_{R-S}|r| d x\right\}^{1 / n},
$$

and condition (4.4) will thus certainly be satisfied if (4.2) holds. This completes the proof of Theorem 4.1 .

To show that the constant in (4.2) is the best possible, we consider the equation

$$
\sigma^{(2 m)}-(-1)^{m} \sigma=0
$$

on the interval $[0, \pi / 2]$. The solution $\sin x$ of this equation, as well as all its derivatives, vanish at either 0 or $\pi / 2$, and the equation is thus not disfocal on $[0, \pi / 2]$. On the other hand,

$$
\max _{c} c^{n-1} \int_{c}^{\pi / 2}|r| d x=\max _{c} c^{n-1}\left(\frac{\pi}{2}-c\right)=(n-1)^{n-1}\left(\frac{\pi}{2 n}\right)^{n}, \quad(n=2 m)
$$

and this shows that the constant in condition (4.2) (which in this case is equivalent to condition (4.3)) cannot be improved upon.

5. Finally, we give a simple example which illustrates the use of Theorem 2.2. We take $B$ to be a matrix whose only nonzero elements $b_{i k}$ appear in the $n$th column, and we set $b_{1, n}=b_{2, n}=\cdots=b_{m, n}=\varepsilon(1 \leqq m \leqq n-1)$, where $\varepsilon$ is a small positive number, and $b_{m+1, n}=\cdots=b_{n, n}=0$. We have $\|B\|=\varepsilon \sqrt{ } m$, and it is easily confirmed that the system (2.2) associated with this matrix $B$ has the solution vector $w=\left(\varepsilon\left(x-x_{1}\right), \varepsilon\left(x-x_{2}\right), \ldots, \varepsilon\left(x-x_{m}\right), c_{m+1}, \ldots, c_{n-1}, 1\right)$, where $x_{1}, \ldots, x_{m}, c_{m+1}$, $\ldots, c_{n-1}$ are arbitrary constants. If $x_{r} \in[a, b], r=1, \ldots, m$, the first $m$ components of $w$ have zeros in $[a, b]$, and Theorem 2.2 can be applied if $y=\left(y_{1}, \ldots, y_{n}\right)$ is a solution vector of $(2.1)$ whose components $y_{m+1}, \ldots, y_{n}$ vanish at points of $[a, b]$. For $\varepsilon \rightarrow 0$, we have $\|B\| \rightarrow 0$ and $v=w /\|w\|$ tends to a constant unit vector $v=\left(v_{1}, \ldots, v_{n}\right)$ with $v_{1}=v_{2}=\cdots=v_{m}=0, v_{k}=\alpha_{k}, k=m+1, \ldots, n$. This leads to the following result.

THEOREM 5.1. Let $y=\left(y_{1}, \ldots, y_{n}\right)$ be a solution vector of the system (2.1). If each of the components $y_{m+1}, \ldots, y_{n}(1 \leqq m \leqq n-1)$ vanishes at some point of the interval $[a, b]$, then 


$$
\left|\arcsin \sum_{k=m+1}^{n} u_{k}(b) \alpha_{k}\right|+\left|\arcsin \sum_{k=m+1}^{n} u_{k}(a) \alpha_{k}\right| \leqq \int_{a}^{b}\|A\| d x
$$

where $u=\left(u_{1}, \ldots, u_{n}\right)=y /\|y\|$, and the $\alpha_{k}$ are such that $\alpha_{m+1}^{2}+\cdots+\alpha_{n}^{2}=1$ and are otherwise arbitrary.

\section{REFERENCES}

1. J. H. Barrett, Fourth order boundary value problems and comparison theorems, Canad. J. Math. 13 (1961), 625-638.

2. E. A. Coddington and N. Levinson, Theory of ordinary differential equations, McGrawHill, New York, 1955.

3. P. Hartman, Ordinary differential equations, Wiley, New York, 1964.

4. P. Hartman and A. Wintner, On disconjugate differential systems, Canad. J. Math. 8 (1956), $72-81$.

5. R. W. Hunt, The behavior of solutions of ordinary, self-adjoint differential equations of arbitrary even order, Pacific J. Math. 12 (1962), 945-961.

6. - Oscillation properties of even-order linear differential equations, Trans. Amer. Math. Soc. 115 (1965), 54-61.

7. W. J. Kim, Disconjugacy and disfocality of differential systems, J. Math. Anal. Appl. (to appear).

8. W. Leighton and Z. Nehari, On the oscillation of solutions of self-adjoint linear differential equations of the fourth order, Trans. Amer. Math. Soc. 89 (1958), 325-377.

9. A. Ju. Levin, Some questions on the oscillation of solutions of linear differential equations, Dokl. Akad. Nauk SSSR 148 (1963), 512-515=Soviet Math. Dokl. 4 (1963), 121-124.

10. - Distribution of the zeros of solutions of a linear differential equation, Dokl. Akad. Nauk SSSR 156 (1964), 1281-1284=Soviet Math. Dokl. 5 (1964), 818-821.

11. D. London and B. Schwarz, Disconjugacy of complex differential systems and equations, Trans. Amer. Math. Soc. 135 (1969), 487-505.

12. Z. Nehari, On an inequality of Lyapunov, Studies in mathematical analysis and related topics, pp. 256-261, Stanford Univ. Press, Stanford, California, 1962.

13. —, Disconjugate linear differential operators, Trans. Amer. Math. Soc. 129 (1967), $500-516$.

14. W. T. Reid, Principal solutions of non-oscillatory self-adjoint linear differential equations, Pacific J. Math. 8 (1958), 147-169.

15. - Oscillation criteria for self-adjoint differential systems, Trans. Amer. Math. Soc. 101 (1961), 91-106.

16. B. Schwarz, Disconjugacy of complex differential systems, Trans. Amer. Math. Soc. 125 (1966), 482-496.

17. T. L. Sherman, Properties of solutions of nth order linear differential equations, Pacific J. Math. 15 (1965), 1045-1060.

18. - Properties of solutions of quasi-differential equations, Duke Math. J. 32 (1965), 297-304.

19. R. L. Sternberg, Variational methods and non-oscillation theorems for systems of differential equations, Duke Math. J. 19 (1952), 311-322.

20. H. M. Sternberg and R. L. Sternberg, A two-point boundary problem for ordinary selfadjoint differential equations of fourth order, Canad. J. Math. 6 (1954), 416-419.

21. A. Wintner, On the non-existence of conjugate points, Amer. J. Math. 73 (1951), 368-380.

\section{CARnegie-Mellon University,} Pittsburgh, Pennsylvania 